SESSION 4 .

OTHER LUMINOUS STARS WITH EMISSION LINES.

Chairman : V. NIEMELA.

1. N.R.WALBORN: Optical and UV Spectral Morphology of Luminous $O B$ Stars in the Galaxy and the Magellanic Clouds.

2. P.S.CONTI: Parameters of Wolf-Rayet Stars.

3. P.MASSEY: Wolf-Rayet Stars in Nearby Galaxies. 
OPTICAL AND ULTRAVIOLET SPECTRAL MORPHOLOGY OF LUMINOUS OB STARS IN THE GALAXY AND THE MAGELLANIC CLOUDS

\author{
Nolan R. Walborn* \\ Space Telescope Science Institute \\ 3700 San Martin Drive \\ Baltimore, MD 21218, U.S.A.
}

\title{
ABSTRACT
}

Three areas of current progress relevant to the theme of this Symposium will be discussed. (1) New spectroscopic observations of the 30 Doradus central cluster, obtained independently by the author and by J. Melnick, confirm the presence of numerous very early o-type members, including several of type 03. In combination with sophisticated new direct imagery of the luminous central object R136 by A. Walker and by $G$. Weigelt, these results have evident implications for understanding the ionization of the supergiant $H$ II region, as well as for the interpretation of R136 itself and of the apparently similar regions seen in more distant galaxies. In particular, no evidence remains for a supermassive object in 30 Doradus, but its central cluster is revealed as a spectacular grouping of very massive hot stars. (2) A further member of the Ofpe/wN9 category in the LMC has been identified, bringing their number to seven, with no exact spectroscopic counterparts yet known in the Galaxy. One of these objects is currently in a state of outburst and has been interpreted by 0 . Stahl et al. as the hottest known Hubble-Sandage variable. (3) An extensive survey of IUE high-resolution data has revealed a strong correlation between the ultraviolet stellar wind features and the optical spectral classifications for the majority of normal 0 stars. These results are relevant to future studies with the High Resolution Spectrograph on the Hubble Space Telescope, which may observe restricted UV wavelength ranges in faint extragalactic OB stars lacking optical data of comparable quality.

\section{THE STELLAR CONTENT OF 30 DORADUS}

There has been substantial progress toward an understanding of the 30 Doradus cluster and its luminous central object R136 since this

\footnotetext{
*Visiting astronomer, Cerro Tololo Inter-American Observatory, which is supported by the National Science Foundation under contract No. AST 78-27879.
} 
subject was reviewed at IAU Symposium 108 (Walborn 1984). Indeed, it is not too strong to say that the situation has developed from one of predominantly indirect, hypothetical, and plausibility arguments at that time, to one dominated by direct evidence now. This evidence shows conclusively that R136 is a complex multiple system, which constitutes the core of a spectacular cluster rich in the most massive hot stars. Important new data have been contributed by Chu, Cassinelli, and Wolfire (1984), Chu and Daod (1984), and Moffat, Seggewiss, and Shara (1985). Qualitatively new information has been provided by two other approaches: sophisticated direct imagery of R136 itself by walker and $O^{\prime}$ Donoghue (1984) and by Weigelt and Baier (1985); and detailed spectroscopy of the cluster stars surrounding R1 36 by Melnick (1985) and by this author (to be presented here).

Walker and O'Donoghue (1984) have analyzed $\mathrm{CCD}$ images of R136 by maximum entropy methods, achieving a resolution of 0.4 and isolating 22 components with $\mathrm{V}$ magnitudes between 11.4 and 15.7 within 4.6 of R136a, including the 0.5 component of $a$ as well as $b$ and $c$. Their outermost faint components are visible in the best direct photographs, such as Figure 3 below. Weigelt and Baier (1985), using the remarkable technique of holographic speckle interferometry, have determined the structure within $\mathrm{R} 136 \mathrm{a}$ at a resolution of $0: 09$, discovering 8 components within 0:70. Two of these components are only 0.3 mag fainter than $a_{1}$ in the red (note that weigelt and Baier have reversed the definition of $a_{2}$ and $a_{3}$ with respect to usage in the previous literature), three are 2 maq fainter, and two more are 2-3 maq fainter. On the assumptions that the visual magnitude differences are the same, that $V=10.8$ for all of R136a (Schmidt-Kaler and Feitzinger 1981), that $A_{v}=1.2$ maq (Panaqia, Tanzi, and Tarenghi 1983; Savage et al. 1983; Fitzpatrick and Savage 1984), and that $\mathrm{v}_{\mathrm{o}}-\mathrm{M}_{\mathrm{v}}=18.6$, it follows that $M_{v}=-7.7$ for $R 136 a_{1},-7.4$ for $a_{2}$ and $a_{3},-5.7$ for $a_{4}-a_{6}$, and -5.2 for $a_{7}-a_{8} \cdot R 136 b$ has $M_{v}=-5.2$ and ${ }^{3}$ has -5.7 . It will be shown below that the same range of values is found for stars in the surrounding cluster. Thus we now have 28 resolved components in $\mathrm{R} 136$ within a radius of $4: 6$, all of which are included in the IUE large aperture as well as in the original optical photometry which was interpreted as due to a single object.

Melnick (1985) has performed an extensive investigation of the spectral types of the 30 Doradus cluster stars surrounding R136. Independently, I have observed about 40 of them with the CTIO 4-meter SIT vidicon system at $1.5 \AA$ resolution, during four nights in Dec. 1984/Jan. 1985. These studies have revealed the most spectacular clustering of massive hot stars for which such detailed information is available. As already indicated by the preliminary results of Melnick (1983), it contains several examples of what may be considered a new class of hot stars, whose spectra show the strong absorption lines of He II and $N$ V and the narrow N IV $\lambda 4058$ emission characteristic of type 03 If*, along with the broad He II $\lambda 4686$ emission typical of $\mathrm{WN}-\mathrm{A}$ spectra. I have classified such spectra as intermediate 03 If*/ WN-A. The first example to be described was Sanduleak $-67^{\circ} 22$, also in 

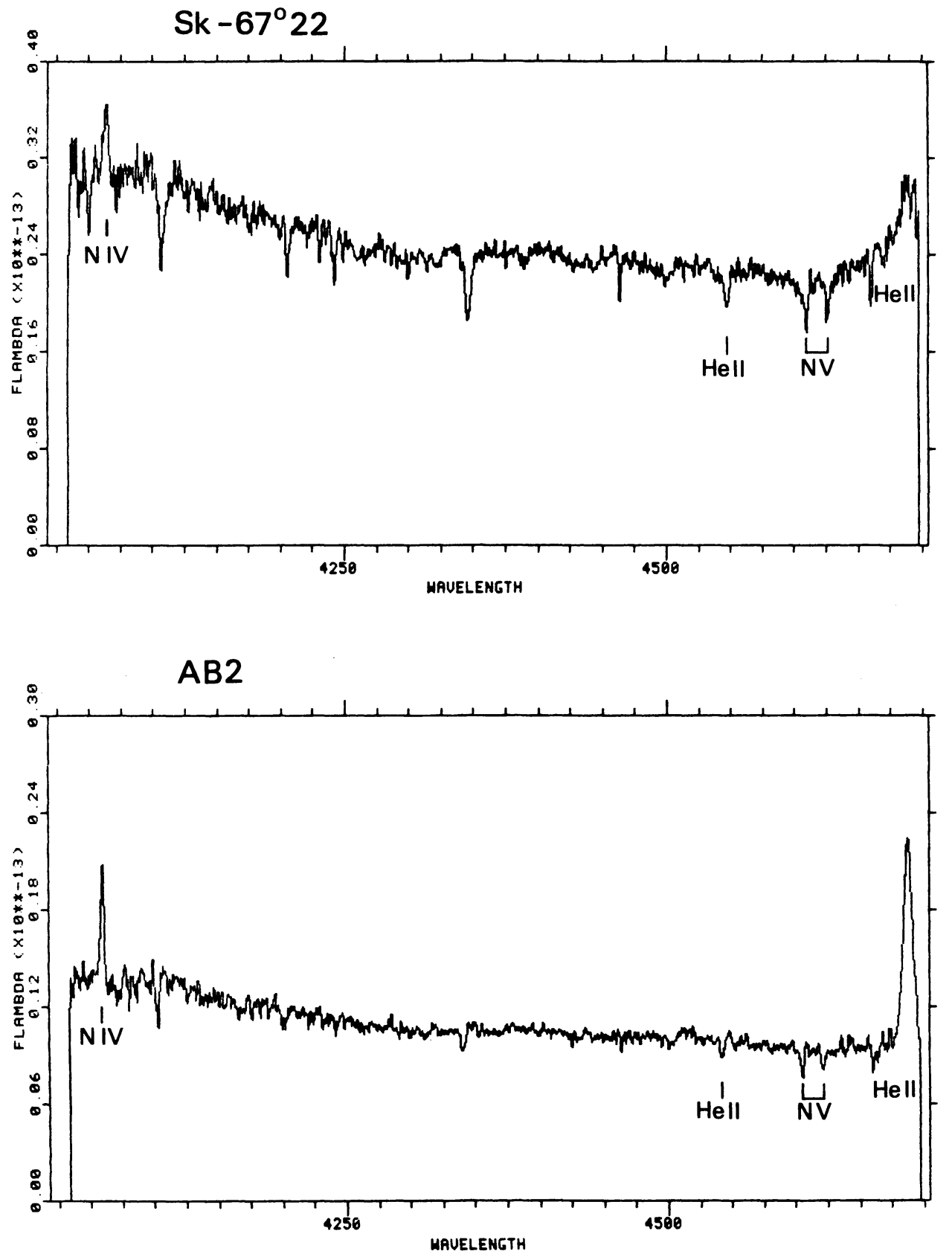

Fig. 1 - Vidicon spectrograms of the $03 \mathrm{If}^{*} / \mathrm{WN} 6-\mathrm{A}$ stars $S k-67^{\circ} 22\left(\mathrm{M}_{\mathrm{v}}=\right.$ $-5.4)$ in the LMC and $A B 2\left(M_{v}=-5.2\right)$ in the SMC. The flux scales are correct as given. The spectral features identified are $N$ IV $\lambda 4058$ emission, He II $\lambda 4541$ and $N$ V $\lambda \lambda$ 4604-4620 absorption, and He II $\lambda 4686$ emission. 

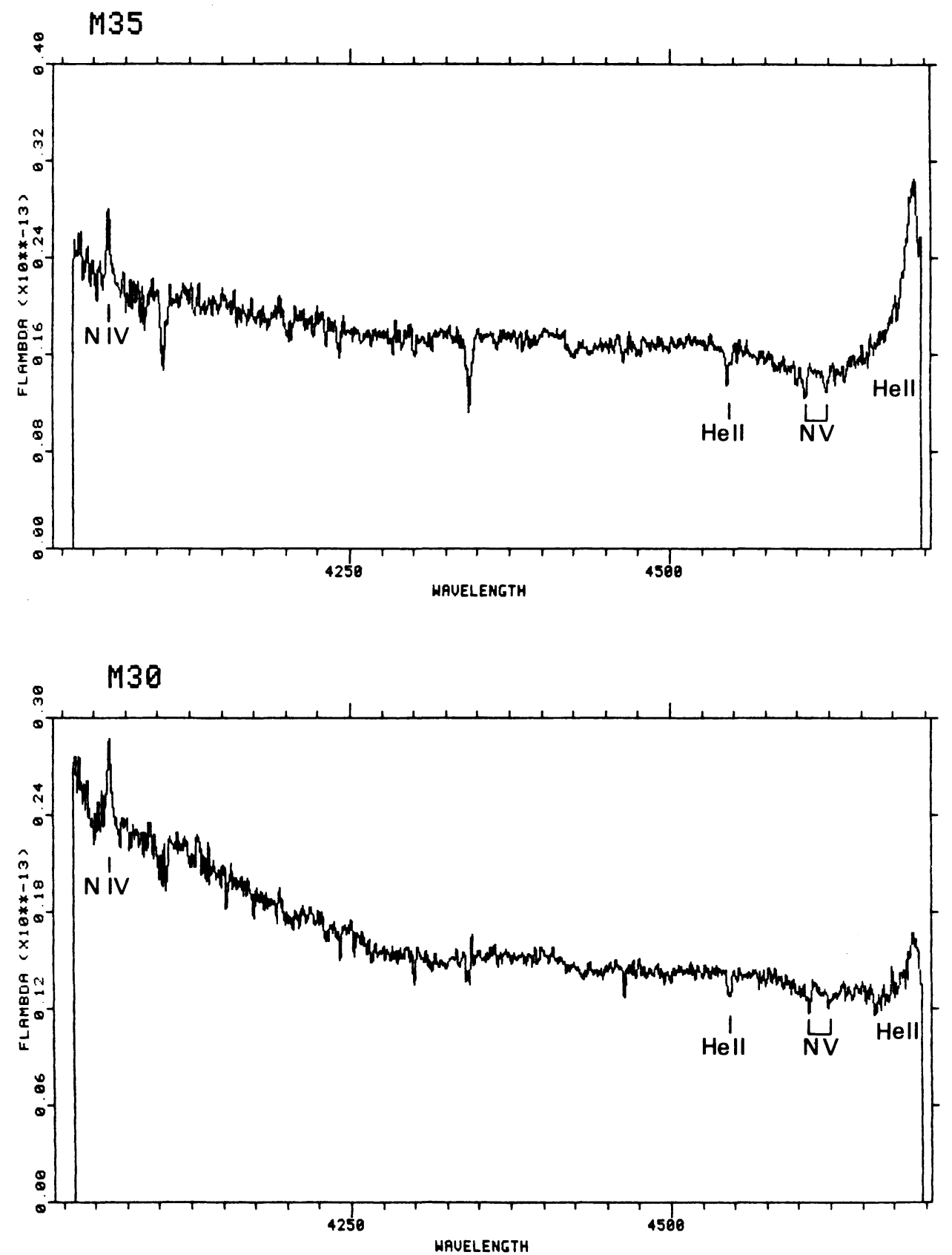

Fig. 2 - Vidicon spectrograms of the 03 If*/WN6-A stars Melnick 30 and 35 in 30 Doradus. The flux scale for M30 should be brighter by a factor of 1.9 at $4330 \AA$ and that for M35 by 1.45. The spectral features identified are as in Fiqure 1. 
the IMC (Walborn 1982a); recently another has been found in the SMC, namely AB2 (Azzopardi and Breysacher 1979a; Garmany and Massey 1984). The vidicon spectrograms of these two stars are shown in Fiqure 1, and those of the similar stars Melnick 30 and 35 in 30 Doradus are shown in Fiqure 2.

Table 1 gives spectral classifications for 56 stars in the 30 Doradus cluster exclusive of $\mathrm{R} 136$, from either my results (W) or Melnick's (M); there is excellent agreement in detail between the two studies. Many of my classifications are preliminary, because the intended tailored nebular emission-line subtractions have not yet been accomplished; in these cases I have given spectral-type ranges which are unlikely to be exceeded. The table contains 15 definite or potential 03 stars, whereas only 12 members of this class were previously known (the 10 discussed by walborn 1982a plus R136a and $A B 2)$. None of the spectral types earlier than 06 was known before these investigations, confirming the suggestion of Melnick (1983) and walborn (1984) that the principal sources of ionization in 30 Doradus other than R136 had not yet been identified. Table 1 also lists blue magnitudes derived from the vidicon fluxes; these have had to be corrected, with reference to the photographic photometry of Westerlund (private communication), for an improper positioning of a guide probe into the telescope beam affecting three of the four nights, and they are accurate to a few tenths of a magnitude. Accurate photometry of the 30 Doradus cluster is in preparation by Melnick and by Chu and Seitzer. The absolute visual maqnitudes in Table 1 are approximate

Table 1. The Stellar Content of 30 Doradus (Exclusive of R136)

Melnick/ Radcliffe

Spectral Type source

$\begin{array}{ll}\text { O3 } & \text { If } * / W N 6-A \\ \text { O3 } & \text { If } * / W N 6-A \\ \text { O3 } & \text { If } * / W N 6-A \\ \text { O3 } & \text { If } * / W N 6-A \\ \text { O3 } & \text { If } * / W N 7-A\end{array}$

$13 \quad 03-4$

23

$03-4$ (f?)

$24 \quad 03-4 \mathrm{~V}$

$25 \quad 03-4 \mathrm{~V}$

26 03-4 V

36 03-4 V

10 03-6 V

14 03-6 V

$15 \mathrm{~S} \quad 03-6 \mathrm{~V}$

55 03-6 V

$m_{B}$

$\begin{array}{ll}13.3 & -6.6 \\ 13.4 & -6.5 \\ 12.6 & -7.3 \\ 12.6 & -7.3 \\ 14.0 & -5.9\end{array}$

$13.9-6.0$

$14.3 \quad-5.6$

$14.0 \quad-5.9$

$12.9 \quad-7.0$

$13.8-6.1$

$14.2-5.7$

$14.2-5.7$

$14.5 \quad-5.4$

$14.3 \quad-5.6$

$14.5 \quad-5.4$ and Shara (1985) 
Table 1. The stellar Content of 30 Doradus (continued)

\begin{tabular}{|c|c|c|c|c|c|}
\hline $\begin{array}{l}\text { Melnick/ } \\
\text { Radcliffe }\end{array}$ & Spectral Type & Source & $m_{B}$ & $\mathrm{M} v$ & Remarks \\
\hline 28 & $04 \mathrm{~V}$ & M & & & \\
\hline 47 & $04-6$ (f) & $\mathrm{W}$ & 13.9 & -6.0 & \\
\hline 4 & $05-6 \mathrm{~V}:$ & $W$ & 14.4 & -5.5 & \\
\hline 8 & $05-6$ & $\mathrm{~W}$ & 14.5 & -5.4 & \\
\hline $35 s$ & $05-6 \mathrm{~V}$ & W & 13.8 & $-6 \cdot 1$ & \\
\hline $35 \mathrm{~N}$ & 05-7: & $\mathrm{W}$ & 14.0 & -5.9 & \\
\hline 48 & $05-7$ & $\mathrm{~W}$ & 14.4 & -5.5 & \\
\hline R139 & 06-7 Iaf & $\mathrm{W}$ & 12.1 & -7.8 & \\
\hline 7 & $O(7) \mathrm{V}$ & M & & & \\
\hline 15 & $07 \mathrm{v}$ & $\mathrm{W}$ & 13.5 & $-6 \cdot 4$ & \\
\hline 21 & $07 \mathrm{~V}$ & M & 14.2 & $-5 \cdot 7$ & \\
\hline R133 & 07-8 II & $\mathrm{W}$ & 12.6 & $-7 \cdot 3$ & \\
\hline 32 & 08 II & $\mathrm{W}$ & 13.1 & -6.8 & \\
\hline 6 & $08-9$ & $\mathrm{~W}$ & 14.6 & $-5 \cdot 3$ & \\
\hline 58 & $08-9$ & W & 13.9 & -6.0 & \\
\hline 59 & $08-9$ & $\mathrm{~W}$ & 14.4 & -5.5 & \\
\hline is & $09-9.5$ & $\mathrm{~W}$ & 14.7 & -5.2 & $\begin{array}{l}90 " \text { south of } \\
\text { Melnick } 1\end{array}$ \\
\hline 38 & $09.7 \mathrm{Iab}$ & W & 13.6 & $-6 \cdot 3$ & Could be OC \\
\hline $14 \mathrm{~N}$ & $\mathrm{OV}$ & M & & & \\
\hline $22 A B$ & 0 & M & 13.8 & $-6 \cdot 1$ & \\
\hline 33 & WC5+O4 & M & & & \\
\hline R140N & WC5+WN4 & M & & & \\
\hline 34 & WN 4.5 & M & & & \\
\hline 37 & WN7-A & $\mathrm{W}$ & 12.7 & -7.2 & $\begin{array}{l}\text { strong, symmetri- } \\
\text { cal Hy absorption }\end{array}$ \\
\hline 49 & WN7 & $\mathbf{M}$ & & & \\
\hline 53 & WN8 & M & & & $\begin{array}{l}\text { Azzopardi and } \\
\text { Breysacher }(1979 b)\end{array}$ \\
\hline R1 34 & WN7 & M & & & \\
\hline R1 35 & WN6 & M & & & \\
\hline R140S & WN 4.5 & M & & & \\
\hline R1 45 & WN6 & M & & & \\
\hline 27 & BO I & $w$ & 13.5 & -6.4 & \\
\hline 50 & BO: I: & W & 14.3 & -5.6 & \\
\hline 11 & $\mathrm{BO}-0.5 \mathrm{Ia}$ & $\mathrm{w}$ & 13.2 & -6.7 & \\
\hline 12 & $\mathrm{BO}-0.5 \mathrm{Ia}$ & W & 12.4 & $-7 \cdot 5$ & \\
\hline 54 & $\mathrm{BO} .5 \mathrm{Ia}$ & W & 13.0 & -6.9 & \\
\hline R1 41 & $\mathrm{~B} 0.5 \mathrm{I}$ & W & 12.4 & $-7 \cdot 5$ & \\
\hline R142 & $\mathrm{B} 0.5-0.7 \mathrm{I}$ & W & 11.8 & $-8 \cdot 1$ & \\
\hline R1 37 & B0.7-1.5 I & W & 12.0 & -7.9 & \\
\hline 52 & B1 Ia & W & 13.8 & -6.1 & \\
\hline 5 & B2: Ip? & W & 14.3 & -5.6 & Hy P Cyg profiles? \\
\hline R138 & AO I & W & & & \\
\hline
\end{tabular}


and have been derived with the assumptions of $B-V=0.1, A_{y}=1.2$, and $v_{o}-M_{v}=18.6$ for all stars. The absolute magnitudes of Melnick 39 and 42 are essentially the same as those of $R 136 a_{1}-a_{3}$.

The distribution of the (abbreviated) spectral types is shown on photographs of the 30 Doradus cluster in Figure 3 for types 03-06 and WR, and in Figure 4 for the later types. Note that four of the 03 If*/WN-A objects immediately surround R136. On the other hand, the later-type stars show rather less of a concentration toward R136. Whether the several late $O$ and early $B$ supergiants present belong to the 30 Dor cluster or are related to the general field in which the nebula is situated will have to be determined by comparably detailed spectroscopy of the latter.

The brightest unresolved component in R136, namely $a_{1}$, is about 1 mag briqhter in $M_{v}$ than HD 93129A; following the discussion of Walborn (1984), the upper limit to its mass is therefore $250 M_{6}$. The entire 30 Doradus cluster, including R136, may contain 15-20 stars with masses of $100-200 \mathrm{M}_{Q}$, and there is no evidence for the presence of substantially greater masses than these. The nebular ionization halance has been recalculated with the new spectral types by Melnick (1985), with the result that $\mathrm{R} 136$ a contributes about one-third of the ionization and the other cluster stars provide the remainder.

As a footnote to this discussion, I would like to mention the interesting VLA study of the optically obscured, galactic supergiant H II region W49 by Dreher et al. (1984). They have discovered a very luminous, multiple stellar system therein, which may well represent an earlier evolutionary state of those in 30 Doradus and NGC 3603 (Walborn 1973), since it is associated with masers and compact H II regions.

\section{A NEW Ofpe/WN9 STAR IN THE IMC}

A large number of $O B$ supergiants with $\mathrm{P}-\mathrm{Cygni}$ and other emissionline characteristics have been known in the LMC since the pioneering work of Henize (1956) and Feast, Thackeray, and Wesselink (1960). Recently a considerable wealth of information about them at both optical and ultraviolet wavelengths has become available through the work of Shore and Sanduleak (1984) and Stahl et al. (1985 and references therein; see also the review by $B$. Wolf in this volume). I have been interested in the hottest subgroup of these objects, which display peculiar of-like spectra (Walborn 1977, 1982b). They may be further subdivided into three categories: (1) HDE 269858 and Sk-67०266, which have exceptionally narrow Si IV $\lambda 4089, N$ III $\lambda 4097$ absorption lines and expanding nebular shells; (2) HDE 269227, HDF. $269927 \mathrm{C}$, and Bohannan-Epps 381, which appear most stronqly related to the narrow-line WN-A sequence; and (3) HDE 269445, a unique superluminous object with a nearly pure emission-line spectrum in the blue-violet. $\mathrm{HDE} 269858(=\mathrm{R} 127)$ is currently in a remarkable state of 


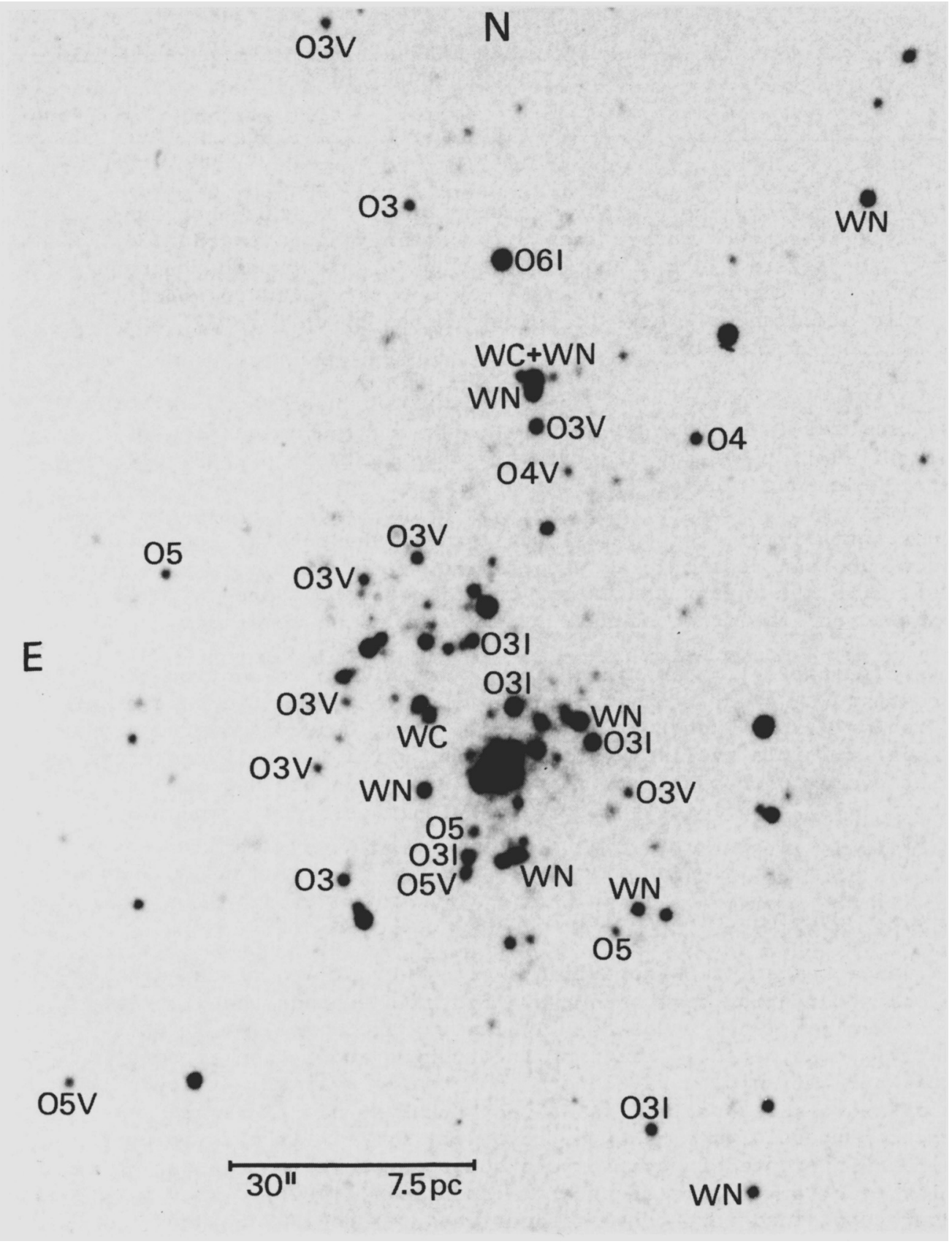

Fig. 3 - The distribution of the 03-06 and WR stars (exclusive of R136) in the 30 Doradus cluster. This excellent photograph was obtained by Dr. Y.-H. Chu through a blue-continuum ( $\lambda 4765)$ interference filter at the CTIO 4-meter prime focus. 


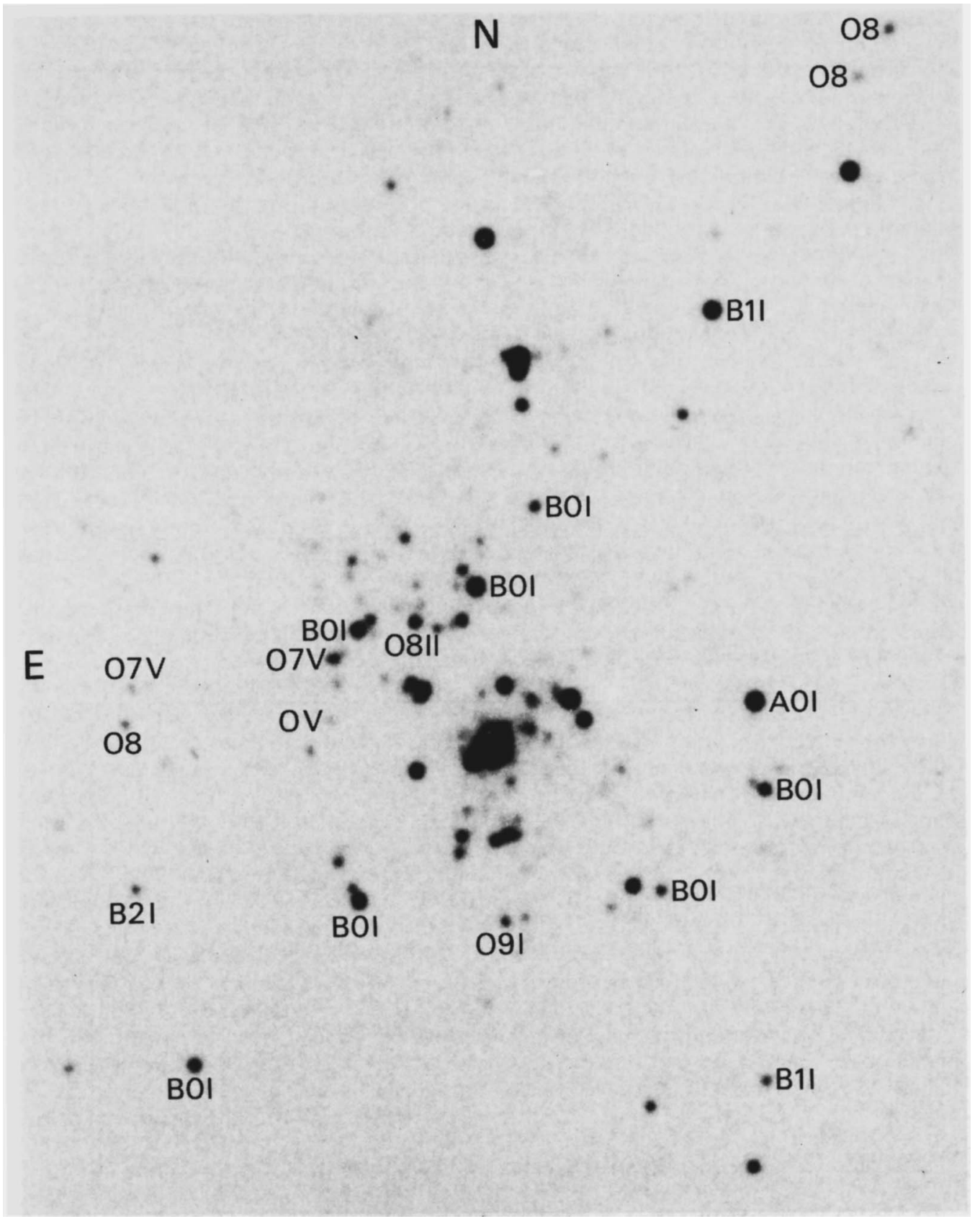

Fig. 4 - The distribution of the later-type stars on an He II $\lambda 4686$ interference-filter photograph of 30 Doradus (which enhances the WR images), also obtained by $\mathrm{Dr}$. Chu at the CTIO 4-meter. 

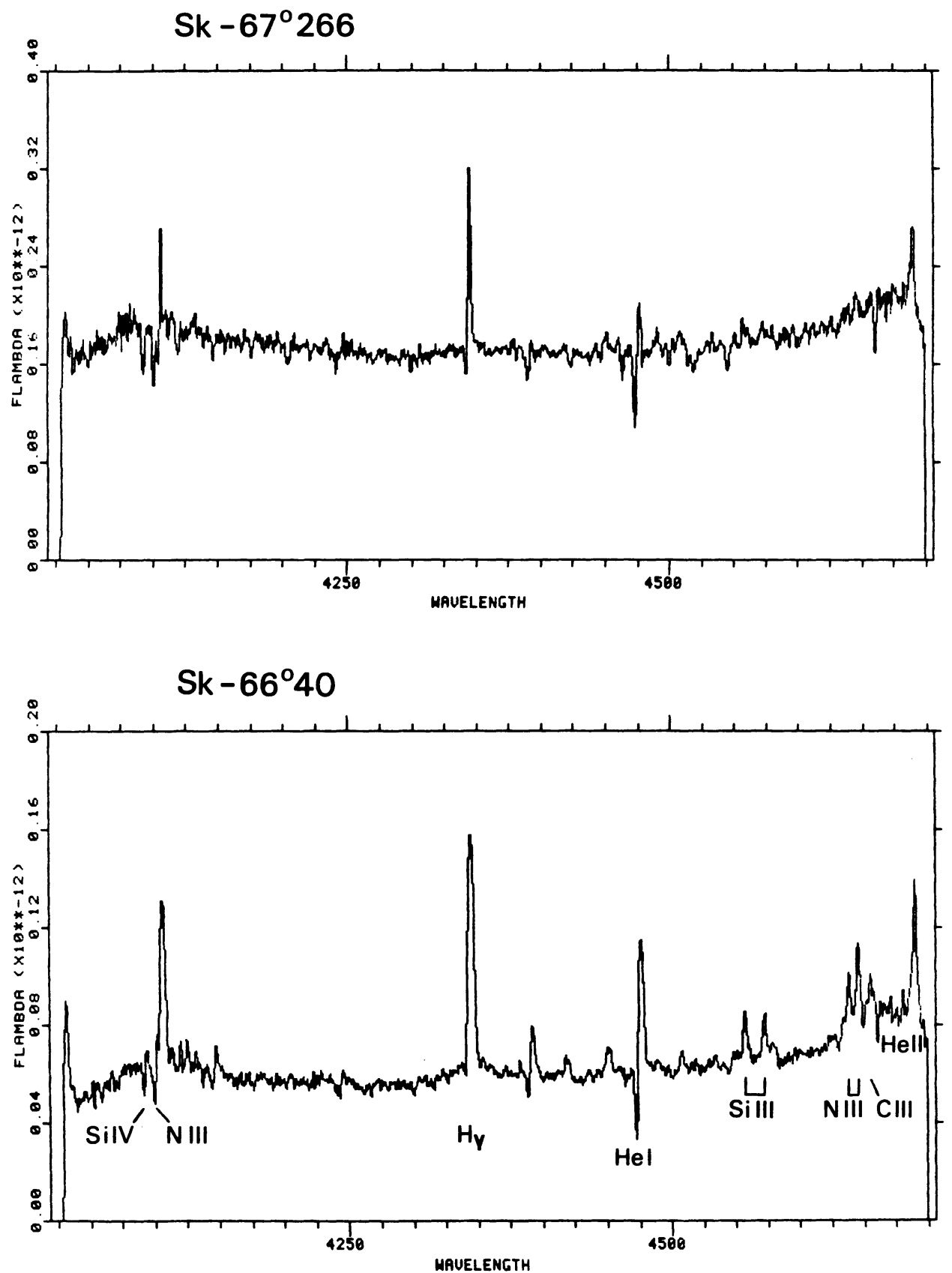

Fig. 5 - Vidicon spectrograms of the ofpe stars $S k-66^{\circ} 40$ and $-67^{\circ} 266$ in the LMC. Both flux scales should be fainter by a factor of 1.7 at $4330 \AA$. The spectral features identified are Si IV $\lambda 4089$ and $N$ III $\lambda 4097$ absorptions, $\mathrm{HY}$ emission, He I $\lambda 4471 \mathrm{P}$ Cygni profile, and Si III $\lambda \lambda 4552-4568, N$ III $\lambda \lambda 4634-4641, C$ III $\lambda 4650$, and HeII $\lambda 4686$ emissions. 
outburst and has been shown to be the hottest known Hubble-Sandage variable by stahl et al. (1983) and Stahl and Wolf (1985); also its nebular shell has apparently been directly resolved by stahl (1985). The purpose of this note is to establish that the star Sk-66\%40 is a further member of the HDE $269858 / \mathrm{Sk}-67^{\circ} 266$ subclass. A SIT vidicon observation of its spectrum is shown along with one of $-67^{\circ} 266$ in Figure 5. The narrow Si IV and N III absorptions are easily seen; the strength of the Si III and C III emission lines in $-66^{\circ} 40$ is also noteworthy. Thus the ofpe/wN9 category in the IMC now contains seven members, while no spectroscopically identical counterparts have yet been identified in the Galaxy.

\section{THE ULTRAVIOLET SPECTRA OF THE O STARS}

Recently an extensive survey of the 1200-1900 $\AA$ region of 0-type spectra has been completed, based upon the unprecedented sample of homogeneous, high-resolution data contained in the International Ultraviolet Explorer archives. The primary result of this survey, comprising about 120 different objects, is conclusive evidence for a high degree of detailed correlation between the UV spectra and the optical spectral classifications, as well as between the photospheric and stellar-wind features, for the great majority of the 0 stars. The pronounced luminosity dependence of the Si IV stellar-wind effect, the main-sequence phenomena, and the oN/OC spectra have been discussed by walborn and Panek (1984a, b; 1985), respectively. The of supergiant and WN-A sequences provide further striking examples of detailed correspondences between stellar-wind features and the optical spectral types. For instance, the $\lambda \lambda 1300-1600 \AA$ region provides a unique signature of an 03 If spectrum, with a strong $O V \lambda 1371$ wind profile, no Si IV $\lambda \lambda 1394-1403$, and very strong C IV $\lambda \lambda 1548-1551$. Similarly, the 04 If spectra, with no $O \mathrm{~V}$, intermediate $\mathrm{Si} I V$, and strong $C$ IV can be readily discriminated from both earlier and later types. Such effects assume considerable significance in the context of future programs with the Hubble Space Telescope High Resolution Spectrograph, since they provide a framework relative to which one can interpret observations of restricted $\mathrm{UV}$ wavelength ranges in faint extragalactic OB spectra lacking high-quality optical data. Unfortunately, space does not permit these spectral sequences to be reproduced here, but an extensive atlas displaying montages of the 1200-1900 $\AA$ range in about 100 objects is currently in press at NASA for wide distribution (Walborn, Heckathorn, and Panek 1985).

\section{REFERENCES}

Azzopardi, M. and Breysacher, J. 1979a, Astron. Astrophys., 75, 120. - 1979b, Astron. Astrophys., 75, 243.

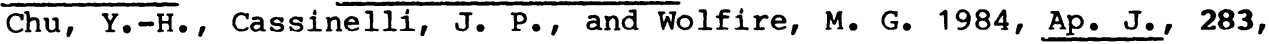
560.

Chu, Y. - H. and Daod, N. A. 1984, P.A.S.P., 96, 999. 
Dreher, J. W., Johnston, K. J., Welch, W. J., and Walker, R. C. 1984, Ap. J., 283, 632.

Feast, M. W., Thackeray, A. D., and Wesselink, A. J. 1960, M.N.R.A.S., $121,337$.

Fitzpatrick, E. L. and Savage, B. D. 1984, Ap. J., 279, 578 .

Garmany, C. D. and Massey, P. 1984, B.A.A.S., 16, 508 .

Henize, K. G. 1956, Ap. J. Suppl., 2, 315.

Melnick, J. 1983, The Messenqer, FSO, No. 32, p. 11. - 1985, Astron. Astrophys., in press.

Moffat, A. F. J., Segqewiss, W., and Shara, M. M. 1985, Ap. J., in press.

Panagia, N., Tanzi, E. G., and Tarenghi, M. 1983, Ap. J., 272, 123.

Savage, B. D., Fitzpatrick, E. L., Cassinelli, J. P., and Fbbets, D. C. 1983, Ap. J., 273, 597 .

Schmidt-Kaler, Th. and Feitzinger, J. V. 1981, in The Most Massive Stars, ed. S. D'Odorico, D. Baade, and K. Kjär (Garching: ESO), p. 105 .

Shore, S. N. and Sanduleak, N. 1984, Ap. J. Suppl., 55, 1.

Stahl, O. 1985, The Messenger, ESO, No. 39, p. 13.

Stahl, O. and Wolf, B. 1985, Astron. Astrophys., in press.

stahl, O., Wolf, B., de Groot, M., and Leitherer 1985, Astron. Astrophys. Suppl., in press.

stahl, O., Wolf, B., Klare, G., Cassatella, A., Krautter, J., Persi, P., and Ferrari-Toniolo, M. 1983, Astron. Astrophys., 127, 49.

Walborn, N. R. 1973, Ap. J. Letters, 182, L21. - 1977, Ap. J., 215, 53. - 1982a, Ap. J. Letters, 254, L15. - 1982b, Ap. J., 256, 452.

- 1984, in IAU Symp. 108, structure and Evolution of the

Magellanic Clouds, ed. S. van den Bergh and $\mathrm{K}$. S. de Boer

(Dordrecht: Reidel), p. 243.

Walborn, N. R., Heckathorn, J. N., and Panek, R. J. 1985,

International Ultraviolet Fxplorer Atlas of O-Type Spectra from 1200 to $1900 \AA$ (NASA), in press.

Walborn, N. R. and Panek, R. J. 1984a, Ap. J. Letters, 280, L27. - 1984b, Ap. J., 286, 718 .

- 1985, Ap. J., 291, 806 .

Walker, A. R. and O'Donoghue, D. E. 1984, Astron. Express, 1, 45. Weigelt, G. and Baier, G. 1985, Astron. Astrophys., in press. 
Discussion : WALBORN.

\section{STALIO :}

Regarding your statement that there exists no individuality among $O B$ stars when you look at the UV lines formed in the stellar wind, I would like to point out the following :

1. That your sample does not include that rather large fraction of $O B$ stars with emission lines : Oe stars and more importantly Be stars. This is a limitation because these stars have the same parameters ( $T$, gravity) as the stars you have studied and there is no reason to invoke high rotation to explain these differences because this is not true (see Polidan, Stalio, Peters, 1985 and references there for a discussion on these points).

2. Costero and I (A.A. Supp1. 58, 95, 1984) found similar morphological results as yours from a smaller sample (40 stars) of nonOe, non-Be stars. However we found measurable differences in edge velocities among stars of the same spectral class. In some cases these differences were quite noticeable. Did you find the same? If yes, how do you explain these differences in terms of current modeling of the stellar wind?

3. I would like to call your attention to a paper by Morton (MNRAS, 189,57, 1979) showing Copernicus OVI profiles of two BOV stars : Tau Sco and upsilon Ori which are absolutely different. IUE unfortunately does not have OVI, but in our poster paper (Polidan and Stalio) we report evidences of changes in OVI in Tau Ori from Voyager data.

4. You didn't mention the occurrences of narrow components in some of these profiles.

\section{WALBORN :}

I did not state that no individuality exists. I stated that the UV spectra of $80-90 \%$ of the 0 stars, including their stellar wind features, display strong systematic trends and a high degree of correlation with their optical spectral types and luminosity classes. They thus provide an empirical reference frame relative to which the 10$20 \%$ of peculiar individuals and categories can be recognized and described.

1. The of stars have enission lines and are included. There are only a handful of known Oe stars. My work does not refer to the $B$ stars. An excellent discussion of the UV spectra of Be relative to 2 . The morphological trends in the wind profiles $I$ have discussed are independent of even large differences in terminal velocity, for the few cases in which the latter occur. For instance, the ON supergiant HD 105056 has an anomalously low terminal velocity, yet displays a supergiant SiIV profile. I have suggested that this star may be less massive than normal stars of the same spectral type (Ap.J. 291, 806, 
1985). Your paper concluded that the UV spectra of the 0 stars on the whole are dominated by individuality, which is incorrect.

3. Tau Sco has highly peculiar, enhanced wind features, which I have discussed in Ap.J. 296, 718 (1984).

4. I did in fact point out narrow absorption components in the CIV profile of $15 \mathrm{Mon}$, and they are reported in a large sample of main sequence spectra in the above references. The variations found in these components and in profile shapes by numerous investigators are small relative to the systematic morphological trends with spectral type and luminosity class.

\section{DE JAGER :}

One of the arguments initially put forward by those, suggesting that R136a should be a supermassive object, was based on the degree of ionization of the HII region surrounding the stellar complex. This would need an object with a temperature of 60000 to $70000 \mathrm{~K}$. This high temperature yields a large bolometric correctign, and that was the only reason for making the luminosity as large as $10^{8}$ solar values. The high temperature and luminosity demands the presence of a large number of 03 objects. Does the observed HII ionization and consequent $\mathrm{T}$ and $\mathrm{L}$ values, agree with the number of 03 objects now identified in that region?

\section{WALBORN :}

Yes. I discussed this question on the basis of photometry in my review at IAU Symposium 108. Melnick (1985, in press) has redone the exercise with the new spectral types; R136 provides one third of the ionization and the other cluster stars provide two thirds. 